\title{
Science Objectives and Initial Operations of SELENE (Kaguya) Mission
}

\author{
By Manabu KATO, Shin-ichi SOBUE, Susumu SASAKI, and Yoshisada TAKIZAWA
}

Japan Aerospace Exploration Agency, Sagamihara, Japan

(Received April 25th, 2008)

\begin{abstract}
The Japanese lunar mission SELENE (Kaguya) was successfully launched by the H2A No.13 rocket on September $14,2007$. After being inserted into a nominal orbit at an altitude of $100 \mathrm{~km}$ for remote sensing on October 19, 2007, a sounder antenna, magnetometer boom, and plasmasphere cameras were deployed before the testing of scientific instruments. Acquisition of scienctific data was carried out for ten months after the nominal mission period that began from the middle of December, 2007. Scientific objectives and perspectives along with the status of the initial operations of Kaguya are described in this paper.
\end{abstract}

Key Words: Kaguya, SELENE, Lunar Science, Scientific Objective

\section{Introduction}

The Apollo missions marked the beginning of the scientific exploration of the Moon. The observation of moonquakes has provided a rough understanding of the lunar interior. The Moon consists of a three-layer structure-crust, mantle, and core ${ }^{1)}$ - which is a common feature of terrestrial planets. Collected rock samples revealed the old formation ages and helped derive a new model for the origin of the Moon. The magma ocean model suggested a "rockberg" floating over a magma ocean during the initial stage of lunar formation around 4.6 billion years $\mathrm{ago}^{2)}$. After a relative lack of lunar missions to the Moon during the 1980s, small robotic missions - the US Clementine ${ }^{3)}$ and Lunar Prospector ${ }^{4)}$ and one Japanese mission-were sent to the Moon. The US missions have observed the entire Moon with respect to elemental and mineral abundances, topography, and gravitational and magnetic fields. The Japanese "Hiten" mission was a technology demonstration mission ${ }^{5)}$. After these precursors, the SELENE (Kaguya) project was promoted to explore the Moon from the viewpoint of lunar studies and future utilization.

\section{Scientific Mission and Onboard Instruments}

Fifteen science instruments ${ }^{6}$, listed in Table 1, were selected for the Kaguya mission after a review process at the Space Science Committee of the Institute of Space and Astronautical Science. These instruments were categorized into five groups depending on their purposes: (1) XRS and GRS to determine the elemental abundance; (2) MI and SP to determine the distribution of mineral abundance, (3) TC, LRS, and LALT to measure the topography of the lunar surface and subsurface; (4) RSAT and VRAD to measure the gravity field of the lunar farside and nearside; and (5) CPS, PACE, RS, and UPI to determine the impact of cosmic radiation and/or solar wind on the Moon and the Earth. In addition, an HDTV was used for public outreach. Each instrument was subjected to a performance test after Kaguya settled into a circular orbit at an altitude of $100 \mathrm{~km}$ on October 19, 2007. The nominal observation began on December 21, 2007, and it lasted for approximately ten months.

Table 1. Science subteams in Kaguya mission.

\begin{tabular}{|l|l|}
\hline Acronyms & Science subteam \\
\hline XRS & X-ray spectrometer \\
\hline GRS & Gamma-ray spectrometer \\
\hline MI & Multiband imager \\
\hline SP & Spectral profiler \\
\hline TC & Terrain camera \\
\hline LRS & Lunar radar sounder \\
\hline LALT & Laser altimeter \\
\hline RSAT & Relay satellite transponder \\
\hline VRAD & Differential VLBI radio source \\
\hline LMAG & Lunar magnetometer \\
\hline CPS & Charged particle spectrometer \\
\hline PACE & Plasma energy, angle and composition experiment \\
\hline RS & Radio science \\
\hline UPI & Upper-atmosphere and plasma imager \\
\hline HDTV & High definition TV camera system \\
\hline
\end{tabular}

\section{Science and Perspectives}

\subsection{Scientific objectives}

All instruments have high-end specifications and they are expected to aid in obtaining valuable data for lunar studies. A particular category of lunar science can be studied comprehensively by integrating data obtained from by several complementary instruments that are used for studying that given category. For example, XRS can be used in combination with GRS. XRS can be used to measure major elements such as $\mathrm{Mg}, \mathrm{Al}$, and $\mathrm{Si}$ with a moderate spatial resolution of $20 \mathrm{~km}$, whereas GRS can be used to measure complementary elements such as $\mathrm{K}$, Th, and $U$ with a low spatial resolution of $100 \mathrm{~km}$. 


\subsection{Chemical constituents of the Moon}

Lunar studies have advanced with the integration of scientific data from various categories. The intention and perspectives of each data from all categories are described in this and the following subsections.

Determining the chemical constituents of the Moon is the first priority from the viewpoint of studying the origin of the Moon and the chemical distribution of the inner zone of the primordial solar system.

Two categories of data, namely, elemental abundance of the lunar surface (obtained by XRS and GRS) and mineral composition (obtained by MI and SP) define the rock types and their distribution on the lunar surface. Information about the subsurface constituents in the lunar crust can be acquired by investigating the central peaks of the craters that were formed by the rebound of impact shock during crater formation. These lunar craters were observed to be greater than $15 \mathrm{~km}$ in diameter. Large basins such as the South Pole-Aitken Basin having a diameter of $2500 \mathrm{~km}$ and a depth of approximately $10 \mathrm{~km}$ expose interior materials of the lower crust or extrude the upper mantle of the Moon. In terms of volume, these remote-sensing data provided information about $15 \%$ of the chemical constituents of the Moon.

In order to estimate the size of the lunar core, gravity-field measurement is used to determine the polar moment of inertia of the Moon. The data obtained by Lunar Prospector was used to determine the iron core radius, which was estimated to be $220-450 \mathrm{~km}^{7}$.

The Kaguya mission provides limited results, and it could not be used to accurately determine the elemental abundance of the entire Moon because it did not contain any instruments for the in situ measurement of the lunar interior. However, it is possible to considerably improve the knowledge about the chemical constituents of the Moon by using data of the constituents of the mantle material by the seismological investigation.

\subsection{Interior structure of the Moon}

As mentioned in the previous subsection, the size of the lunar core could be estimated by the polar moment of inertia of the Moon, which was determined from gravity-field measurements. The shallow interior and subsurface structures could be investigated using LRS. Sounding was carried out using a $5-\mathrm{MHz}$ radio wave to reveal the subsurface layer structure, including the density and/or material discontinuity, up to a depth of approximately $5 \mathrm{~km}$.

Gravity data obtained using RSAT and VRAD and topography data obtained using LALT will be used to estimate the thickness of the entire lunar crust. The crust at the basin area and mares at the lunar nearside is thin, and the highland at the farside is overlaid on a thick crust. The Kaguya mission was successful in determining the crustal thickness with greater accuracy.

\subsection{Dichotomy of nearside and farside of the Moon}

The dichotomy of the Moon is determined from the topography and rock distribution between the lunar nearside and farside. Large mares occupy $60 \%$ of the lunar nearside. A large altitude difference of more than 18 $\mathrm{km}$ was observed in the lunar farside. The dichotomy was determined by the geological study of the material distribution and crustal thickness.

\subsection{Differentiation in magma ocean}

If the Moon did in fact originate from the formation of a magma ocean, evidence of the same must be retained on the lunar surface. The rock distribution could be used as evidence for the differentiation of the magma ocean. The formation of the South Pole-Aitken Basin and large mares due to flooded magma in the nearside are the main geological events that occurred after the formation of the magma ocean 4.6 billion years ago. Therefore, geological recovery or reburying of the basin and mares are necessary to reproduce the magma ocean age. A detailed geological study by the Kaguya mission clarified the origin of magma ocean. Magma ocean model suggests that the Moon formed due to the giant impact. A short duration of accretion to the Moon after the impact led to the heating up of its surface; this was sufficient for the formation of magma.

\subsection{Origin of lunar magnetic field}

Apollo rock sample contains magnetic minerals assuming magnetization in a weak but definite magnetic field. A previous study has indicated that the most probable sources of the ancient lunar magnetic fields were (1) a former core dynamo during a high-field epoch and (2) transient magnetic fields generated by the interaction of impact plasmas with the ambient field during brief periods of ejecta emplacement ${ }^{8)}$. The LMAG Kaguya mission aims at finding weak magnetic remnants less than $10^{-5} \mathrm{~T}$ using an electron reflectometer.

\subsection{Lunar tectonics}

The evolution of the Moon is recorded in various features of the subsurface structure, volcanic activities to 3.2 billion years after origin of 4.6 million.

\section{Summary}

The Kaguya mission will complete the nominal mission period in the end of October, 2008 and follow an optional observation from low altitude orbits using saved fuel.

The ultimate objectives of lunar studies are to determine the origin and evolution of the Moon. Advanced scientific studies such as those mentioned in the previous section may lead to the realization of these objectives. The Kaguya mission is expected to provide new insights for lunar studies.

\section{Acknowledgments}

We would like to send much thanks to member of the Kaguya team for their collaboration. We also thank gratefully anonymous supporters in Japanese planetary science community and international colleagues.

\section{References}

1) Toksoz, M.N., Dainy, A.M., Solomon, S.C., and Anderson, 
K.R.: Velocity Structure and Evolution of the Moon, Proc., $4^{\text {th }}$ LSC (1973), pp. 2529-2547

2) Longhi, J.: Pyroxene Stability and the Composition of the Lunar Magma Ocean, Proc., $9^{\text {th }}$ LPSC (1978), pp. 285-306.

3) Nozette, S., Rustan, P., Pleasance, L.P., Horan, D.M., Regeon, P., Shoemaker, E.M., Spudis, P.D., Acton, C.H., Baker, D.N., Blamont, J.E., Buratti, B.J., Corson, M.P., Davies, M.E., Duxbury, T.C., Eliason, E.M., Jakosky, B.M., Kordas, J.F., Lewis, I.T., Lichtenberg, C.L., Lucey, P.G., Malaret, E., Massie, M.A., Resnick, J.H., Rollins, C.J., Park, H.S., McEwen, A.S., Priest, R.E., Pieters, C.M., Reisse, R.A., Robinson, M.S., Simpson, R.A., Smith, D.E., Sorenson, T.C., Vorder Breugge, R.W., and Zuber, M.T.: The Clementine Mission to the Moon: Scientific Overview, Science, 266 (1994), pp. 1835-1836.

4) Binder, A.B.: Lunar Prospector: Overview, Science, 281 (1998), pp. 1475-1476

5) Uesugi, K.: Results of the MUSES-A “HITEN” Mission, Adv., Space Res., 18 (1996), pp. 69-72
6) Kato, M., Sasaki, S., Tanaka, K., Iijima, Y., and Takizawa, Y.: The Japanese lunar mission SELENE: Science Goals and Present Status, Adv. Space Res., 42 (2008), pp. 292-300.

7) Konopoliv, A.S., Binder, A.B., Hood, L.L., Kuchinskas, A.B., Sjorgen, W.L., and Willliams, J.G..: Improved Gravity Field of the Moon from Lunar Prospector, Science, 281 (1998), pp. 1476-1480.

8) Hood, L.L., Zakharian, A., Halekas, J.,Mitchell, D.L., Lin, R.P., Acuna, M.H., and Binder, A.B.: Initial Mapping and Interpretation of Lunar Crustal Magnetic Anomalies Using Lunar Prospector Magnetometer Data, J. Geophys. Res., 106 (2001), pp. 27825-27839. 